\title{
Über das Sulfat und Oxalat der Scandinerde.
}

\author{
Von \\ F. WIRTH.
}

Mit 2 Figuren im Text.

I.

Über die Löslichkeitsverhältnisse des Sulfats und Oxalats des Scandiums in Säuren, die bei der analytischen Trennung und Reindarstellung dieses Elementes eine große Rolle spielen, sind bisher keine systematischen Untersuchungen ausgeführt worden.

Bezüglich des Sulfats gibt der Entdecker des Scandiums, L. F. NILson ${ }^{1}$, nur an, daB es auBerordentlich leicht in Wasser löslich ist. Nach Crookes ${ }^{2}$ enthalten 100 Teile der bei $12^{\circ}$ gesättigten Lösung 44.5 Teile wasserfreies Scandiumsulfat.

Das Oxalat des Scandiums ist nach diesen Autoren etwas löslich in Säuren und sogar in Wasser. NILSON gibt an, daB $100 \mathrm{ccm}$ einer $1 \%$ igen Schwefelsäure $0.0274 \mathrm{~g} \mathrm{Sc}_{2} \mathrm{O}_{3}=0.0926 \mathrm{~g}$ Oxalathydrat lösen. Nach R. J. MEYeR ${ }^{3}$ bedarf es daher eines großen Überschusses an Oxalsäure, um das Oxalat aus saurer Lösung annähernd vollständig $z u$ fällen. Nach diesem Autor ${ }^{4}$ löst eine $10 \%$ ige Salzsäure beim Siedepunkt 0.8 , eine $20 \%$ ige $1.8 \%$ Oxalathydrat auf.

Nach meinen Versuchen ist bei $25^{\circ}$ allein das 5-Hydrat $\mathrm{Sc}_{2}\left(\mathrm{SO}_{4}\right)_{3} .5 \mathrm{H}_{2} \mathrm{O}$ stabil, das bei etwas höherer Temperatur als $100^{\circ}$ in das Bihydrat $\mathrm{Sc}_{2}\left(\mathrm{SO}_{4}\right)_{3} \cdot 2 \mathrm{H}_{2} \mathrm{O}$ übergeht. Das Pentahydrat ist an der Luft nicht hygroskopisch. Es ist beträchtlich in Wasser löslich, allein schon geringe Mengen freier Schwefelsäure setzen die Löslichkeit ganz enorm herab. Es verhält sich also ganz genau

1 Berl. Ber. 1880, 1430 u. 1439.

2 Z. anorg. Ohem. 61, 349.

${ }^{3}$ Analyse d. selt. Erden u. Erdsäuren 1912, S. 116.

4 Z. anorg. Chem. 67 (1910), 398. 
F. Wirth.

wie das gleichfalls schwach basische Erbiumsulfatenneahydrat. ${ }^{1}$ In nachstehender Tabelle sind die erhaltenen Werte eingetragen.

Tabelle 1.

\begin{tabular}{c|c|c|c}
\hline Bodenkörper & $\begin{array}{c}\text { Lösungsmittel } \\
\text { n. d. } \mathrm{H}_{2} \mathrm{SO}_{4}\end{array}$ & $\begin{array}{c}\text { In } \begin{array}{c}100 \mathrm{~g} \text { Fl. sind gelöst bei } 25^{\circ} \\
\text { Oxyd }\end{array} \\
\mathrm{Se}_{2}\left(\mathrm{SO}_{4}\right)_{3}\end{array}$ \\
\hline $\mathrm{Sc}_{2}\left(\mathrm{SO}_{4}\right)_{3} .5 \mathrm{H}_{2} \mathrm{O}$ & Wasser & 10.23 & 28.27 \\
$\mathrm{Sc}_{2}\left(\mathrm{SO}_{4}\right)_{3} .5 \mathrm{H}_{2} \mathrm{O}$ & Wasser & 10.41 & 28.77 \\
$\mathrm{Sc}_{2}\left(\mathrm{SO}_{4}\right)_{3} .5 \mathrm{H}_{2} \mathrm{O}$ & $1 / 2-\mathrm{n}$. & 10.60 & 29.29 \\
$\mathrm{Sc}_{2}\left(\mathrm{SO}_{4}\right)_{3} .5 \mathrm{H}_{2} \mathrm{O}$ & $1-\mathrm{n}$. & 7.192 & 19.87 \\
$\mathrm{Se}_{2}\left(\mathrm{SO}_{4}\right)_{3} .5 \mathrm{H}_{2} \mathrm{O}$ & 4.86 & 3.026 & 8.363 \\
$\mathrm{Sc}_{2}\left(\mathrm{SO}_{4}\right)_{3} .5 \mathrm{H}_{2} \mathrm{O}$ & 9.73 & 0.476 & 1.315 \\
$\mathrm{Sc}_{2}\left(\mathrm{SO}_{4}\right)_{3} .3 \mathrm{H}_{2} \mathrm{SO}_{4}$ & 22.35 & 0.175 & -
\end{tabular}

Von den bisher untersuchten Sulfaten der seltenen Erden be. sitzt also das Scandiumsulfat die gröBte Löslichkeit in Wasser. Nimmt man für die Wasserlöslichkeit den Mittelwert, so sind in $100 \mathrm{~g}$

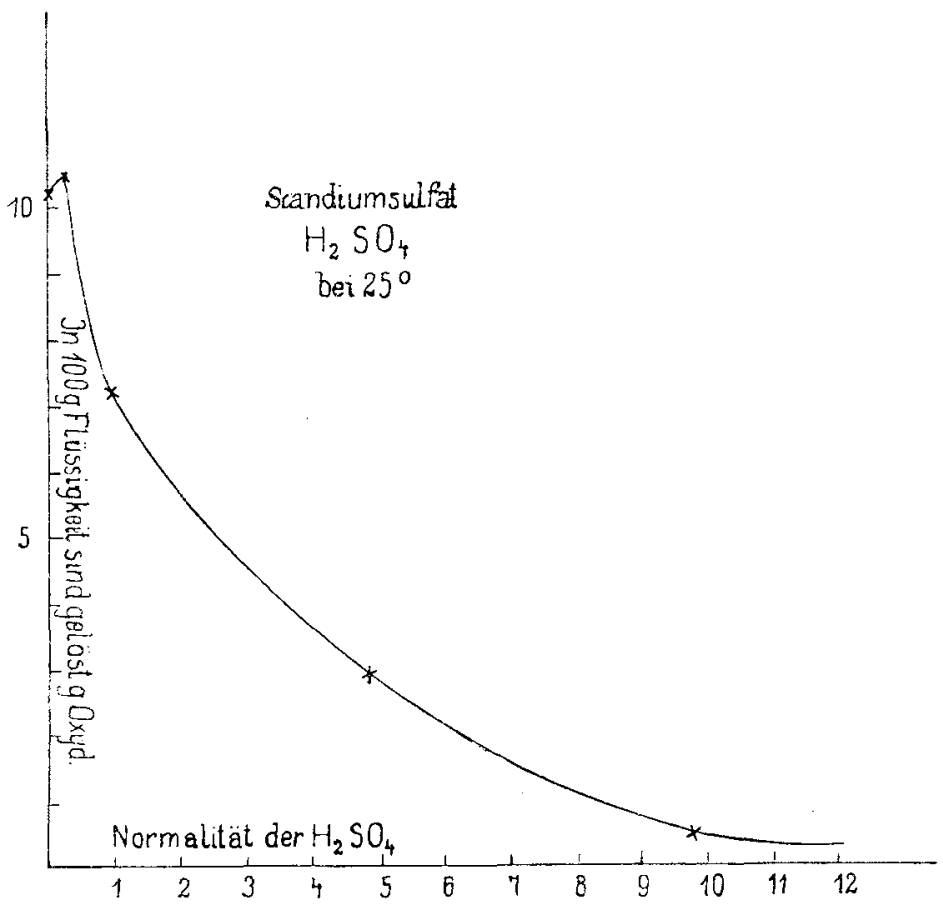

Flüssigkeit $28.52 \mathrm{~g} \mathrm{Sc}_{2}\left(\mathrm{SO}_{4}\right)_{3}=35.32 \mathrm{~g} \mathrm{Sc}_{2}\left(\mathrm{SO}_{4}\right)_{3} .5 \mathrm{H}_{2} \mathrm{O}$ gelöst. $100 \mathrm{ccm}$ Wasser Jösen also bei $25^{\circ} 54.61 \mathrm{~g}$ Scandiumsulfatpentahydrat auf. Über die Löslichkeit derSulfate von den Spaltungsprodukten des alten

1 F. Wirte, Z. anorg. Chem. 76 (1912), 174. 
Ytterbiums liegen noch keine exakten Angaben vor, doch scheint das Ytterbium im allgemeinen sehr leicht lösliche Salze zu bilden. Die Löslichkeit des Erbiumsulfats in Wasser ist bedeutend geringer In $100 \mathrm{~g}$ Flüssigkeit sind bei $25^{\circ}$ nur $11.94 \mathrm{~g} \mathrm{Er}_{2}\left(\mathrm{SO}_{4}\right)_{3}$ gelöst. $^{7}$

\section{II.}

Das Oxalat des Scandiums fällt aus schwach saurer Lösung mit Oxalsäure als schlecht kristallisiertes Pentahydrat $\mathrm{Sc}_{2}\left(\mathrm{C}_{2} \mathrm{O}_{4}\right)_{3} .5 \mathrm{H}_{2} \mathrm{O}$ aus, das bei ca. $120^{\circ}$ glatt in das Monohydrat übergeht. In schön kristallisierter Form erhält man es, wenn eine heiß gesättigte Lösung des Oxalats in Ammonoxalat durch Salzsäure zersetzt wird.

Ich bestimmte vorläufig die Löslichkeit des Oxalats in Schwefelsäuren verschiedener Konzentration bei $25^{\circ}$. Tabelle 2 und Fig. 2 enthalten die Resultate.

Tabelle 2.

\begin{tabular}{|c|c|c|}
\hline Normalität der $\mathrm{H}_{2} \mathrm{SO}_{4}$ & 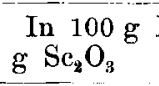 & $\begin{array}{l}\text { nd gelöst: } \\
\mathrm{g} \mathrm{Sc}_{2}\left(\mathrm{C}_{2} \mathrm{O}_{4}\right)_{3}\end{array}$ \\
\hline 1 & 0.0444 & 0.1148 \\
\hline 2.1 & 0.0995 & 0.2573 \\
\hline 2.43 & 0.1132 & 0.2904 \\
\hline 3.57 & 0.1625 & 0.4202 \\
\hline 4.32 & 0.2008 & 0.5192 \\
\hline 4.86 & 0.2256 & 0.5834 \\
\hline
\end{tabular}

Das Oxalat der Scandinerde ist also von allen bisher untersuchten Oxalaten der seltenen Erden am schwersten in Schwefelsäure löslich. Wendet man z. B. eine 4.32-norm. $\mathrm{H}_{2} \mathrm{SO}_{4}$ an, so sind

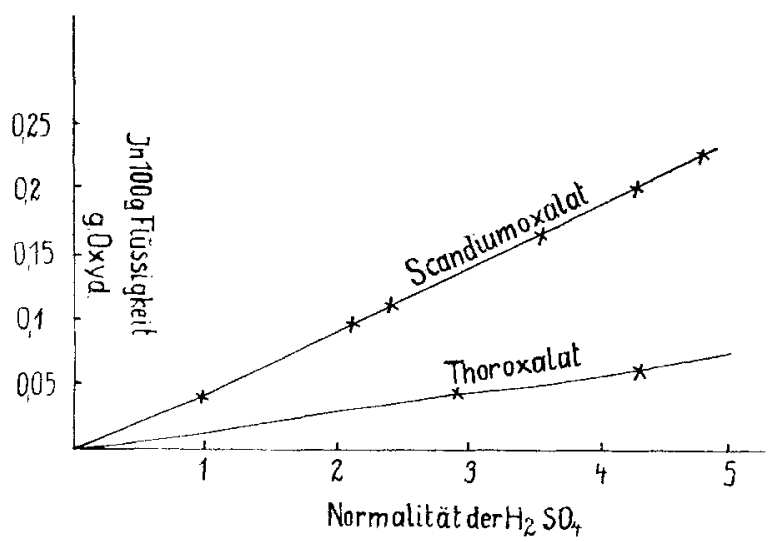

Fig. 2.

${ }^{1}$ F. Wirth, $Z$. anorg. Chem. 76 (1912), 174. 
bei $25^{\circ}$ in $100 \mathrm{~g}$ Flüssigkeit gelöst: $0.88 \mathrm{~g} \mathrm{La}_{2} \mathrm{O}_{3}, 0.75 \mathrm{~g} \mathrm{CeO}_{2}$, $0.7 \mathrm{~g} \mathrm{Er}_{2} \mathrm{O}_{3}, 0.44 \mathrm{Gd}_{2} \mathrm{O}_{3}, 0.43 \mathrm{Sm}_{2} \mathrm{O}_{2}, 0.20 \mathrm{Sc}_{2} \mathrm{O}_{3}$ und $0.11 \mathrm{ThO}_{2}$. Die Löslichkeitswerte des Scandiums nähern sich mebr den bei der Untersuchung des Thoroxalats ${ }^{1}$ gefundenen Zahlen, die ebenfalls in Fig. 2 eingetragen wurden.

Um den Einfluß von überschüssiger Oxalsäure zu ermitteln, wurde Scandiumoxalat 12 Tage bei $25^{\circ}$ mit einer Lösung geschüttelt, die bezüglich der $\mathrm{H}_{2} \mathrm{SO}_{4}=2.43$-norm., bzw. der Oxalsäure $=1 / 2$-norm. war. In $100 \mathrm{~g}$ Flüssigkeit wurden $0.0284 \mathrm{~g} \mathrm{Sc}_{2} \mathrm{O}_{3}$ gefunden, während in $100 \mathrm{~g}$ einer 2.43-norm. Schwefelsäure, der keine überschüssige Oxalsäure zugesetzt ist, $0.1123 \mathrm{~g} \mathrm{Sc}_{2} \mathrm{O}_{3}$ gelöst sind. Die Löslichkeitserniedrigung beträgt also in diesem Falle $=74.71 \%$.

${ }^{1}$ F. WiRTH, Z. anorg. Chem. 76 (1912), 174.

Berlin, Anorg.-chem. Institut der Kgl. Techn. Hochschule.

Bei der Redaktion eingegangen am 14. März 1914. 